\title{
Capillary filling of miniaturized sources for electrospray mass spectrometry
}

\author{
Steve Arscott ${ }^{1}$, Matthieu Gaudet ${ }^{1}$, Martin Brinkmann ${ }^{2,3}$, \\ Alison E Ashcroft ${ }^{4}$ and Ralf Blossey ${ }^{2}$ \\ ${ }^{1}$ Institut d'Electronique de Microélectronique et de Nanotechnologie (IEMN), CNRS UMR 8520, \\ Avenue Poincaré BP 60069, F-59652 Villeneuve d'Ascq, France \\ 2 Biological Nanosystems Group, Interdisciplinary Research Institute (IEMN), Avenue Poincaré \\ BP 60069, F-59652 Villeneuve d'Ascq, France \\ ${ }^{3}$ Max-Planck Institute of Dynamics and Self-Organization, Bunsenstrasse 10, 37073 Göttingen, \\ Germany \\ ${ }^{4}$ Astbury Center for Structural Molecular Biology, University of Leeds, Leeds LS2 9JT, UK
}

\begin{abstract}
Capillary slot-based emitter tips are a novel tool for use in electrospray ionization-mass spectrometry of large biomolecules. We have performed a combined theoretical and experimental study of capillary filling in micron-sized slots with the aim of developing a rational design procedure for miniaturized electrospray sources, ultimately enabling the integration of ESI into laboratoryon-a-chip devices.
\end{abstract}

\section{Introduction}

Today, the applications of electrically charged liquid droplets cover a wide spectrum which extends all the way from painting and ink-jet printing to mass spectrometry. One method for the production of electrically charged liquid droplets is that of electrospraying, i.e. the break-up of a liquid drop by the application of a high electric field. The phenomenon of electrospraying was first studied by John Zeleny at the University of Minnesota in the early part of the 20th century [1,2]. Later, Seville Chapman, whilst working at Berkeley in the 1930s, generated an electrospray using a glass sprayer capillary tube with a voltage generator in the range $\pm 4.6 \mathrm{kV}$ [3]. In the 1960s, Malcolm Dole and colleagues at Northwestern University were the first to produce a molecular beam of macro-ions using a stainless steel hypodermic needle (Hamilton No N792) with a 'bevelled tip having a length of 1 inch and an inside diameter of 8 mils'; 8 mils is equivalent to approximately $200 \mu \mathrm{m}[4,5]$. Dole et al showed conclusively that distinct macro-ions of definite mass-charge states could be produced in the gaseous state by electrospray techniques. Following the work by the Dole group, it was John Fenn et al who, at Yale in the 1980s, applied electrospraying for the formation of charged droplets for mass spectrometry applications [6-8]. Fenn et al initially produced an electrospray ion source in 
the voltage range $3-10 \mathrm{kV}$, again using a stainless steel hypodermic needle (B\&S gauge 26s) having a smaller inside diameter of $100 \mu \mathrm{m}$ as compared to the Dole group.

The electrospray process has profoundly affected the field of mass spectrometry by allowing the structural analysis of virtually unlimited molecular weights, e.g., large biomolecules. The concept of a nanoelectrospray (nanoESI) was introduced by Wilm and Mann [9]. Nanospray, a soft ionization technique, is defined by a low voltage operation

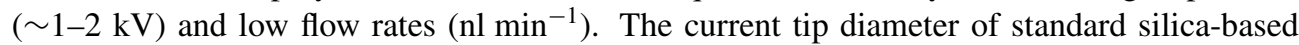
needle-type electrospray sources is of the order of 1-5 $\mu \mathrm{m}$ [9]. However, by both reducing the size of the electrospray emitter tip to micron and sub-micron dimensions and producing reproducible tip-to-tip dimensions via the use of micro- and nanotechnology there is still much to be gained in terms of the physical understanding of the electrospray itself and how the electrospray relates to the resultant mass spectra. In addition to this, one should not forget the benefits in terms of the potential integration with laboratory-on-a-chip (LOC) systems.

Although a number of micro-machined ESI sources have already been described in the literature [10-15], we have recently proposed an original electrospray source based on a microfluidic capillary slot [16] and a nanofluidic capillary slot [17, 18]. Moreover, we have presented design rules for capillary slot-based electrospray sources [19].

In this work we discuss the physics of spontaneous capillary slot filling by a liquid. We introduce, for the first time, the phenomenon of meniscus overshoot at the end of the capillary slot and study it both theoretically and experimentally.

The plan of the paper is as follows. We first present the basic design of the electrospray sources, together with a brief review of their fabrication using microtechnology. Then, we turn to the theoretical description of capillary slot filling and introduce the meniscus overshoot. Microfluidic experiments to test theoretical predictions follow, and we also present some results from the application of the sources for mass spectrometry. The paper ends with a discussion of further directions of research.

\section{Capillary slot-based electrospray sources}

\subsection{Device topology and fabrication}

The originality of the nanoESI MS source is the open topology, which is a capillary slot rather than a capillary tube; figure 1 shows an example. The sources are composed of the following elements: a silicon support wafer and a polysilicon layer in which the following features are defined: a reservoir and a capillary slot having a length $L$, a width $w$ and a height $h$ formed by two polysilicon cantilevers which project horizontally beyond the edge of the silicon wafer. This topology enables easy coupling of the chip to the input of a mass spectrometer. The reservoir contains the sample test liquid while the capillary slot leads the test liquid along the slotted cantilever by capillary action from the reservoir to the source tip. The source is then placed at the inlet of the mass spectrometer (MS) for testing. The high voltage (HV) required for electrospraying is applied to the semiconducting silicon wafer, thus enabling a low resistance path to the liquid in the reservoir. The triangular shaped cantilevers which form the point-like feature necessary for nanoESI are fabricated from polysilicon having a thickness of a few $\mu \mathrm{m}$ generally. The slotted polysilicon-based cantilevers form a capillary slot having dimensions of $w \times h$.

The topology of the interface presented here requires long rigid triangular cantilevers which form a well defined microfluidic capillary slot over the whole length of the source which is intended to bring a liquid to a point. The requirement here of a long, point-like structure implies that polysilicon is an excellent choice as the Young modulus of as-grown polysilicon has been measured to lie between 120 and $200 \mathrm{GPa}$. 


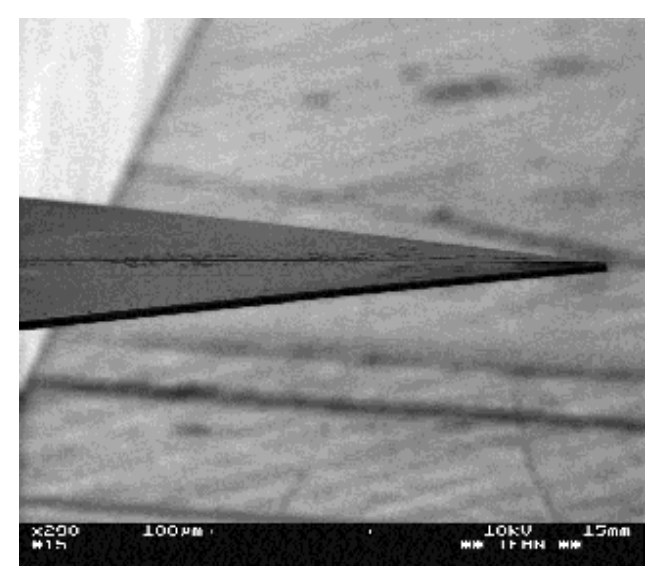

Figure 1. A micronib electrospray emitter tip composed of two triangular polysilicon cantilevers forming a central capillary slot having a slot width $w$ and height $h$ of micron sized dimensions.

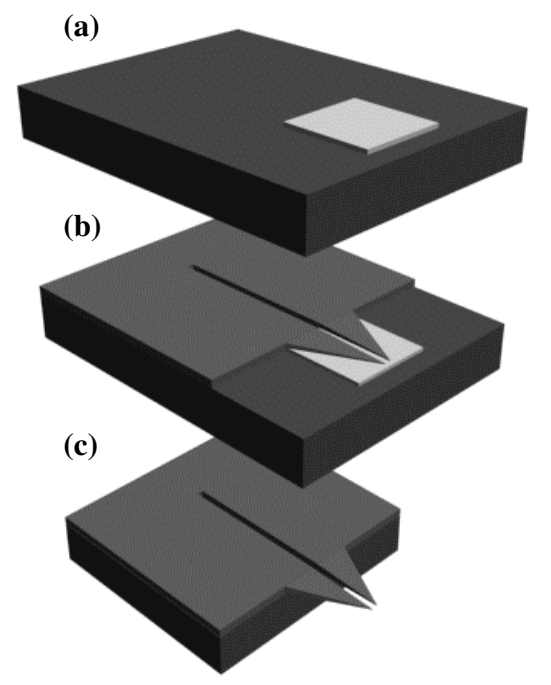

Figure 2. Three step microfabrication process used to make the capillary slot-based electrospray sources: (a) deposition and patterning of sacrificial etch layer (white), (b) deposition and definition of micronib (light grey) and (c) under-etch of sacrificial layer followed by nib release from wafer.

Figure 2 illustrates the silicon-based micro-machining process which has been developed for the fabrication of the polysilicon based cantilever nanoESI sources. This process consists of three main technological steps: (i) sacrificial etch release layer deposition/patterning, (ii) low-stress polysilicon deposition/patterning and (iii) wafer cleaving. A 3-inch diameter n-type doped silicon wafer having a (100) orientation was employed for the fabrication of the cantilever structures. Firstly, a $\mathrm{SiO}_{2}$ thermal oxide $(200 \mathrm{~nm})$ layer is grown by dry oxidation in a furnace. This oxide serves as the sacrificial layer for the release of the slotted cantilevers from the silicon wafer surface.

The thermal $\mathrm{SiO}_{2}$ layer is subsequently patterned in order to form the mesa features which act as the sacrificial etch release layers underneath the polysilicon cantilevers. A layer of nondoped polycrystalline silicon $(2-5 \mu \mathrm{m})$ which defines $h$ is deposited onto the wafer using 
low pressure chemical vapour deposition (LPCVD) by the thermal decomposition of silane $\left(\mathrm{SiH}_{4}\right)$. In order to minimize the internal stresses in this layer, a high temperature $\left(1050{ }^{\circ} \mathrm{C}\right)$ anneal is performed. A low temperature silicon dioxide (LTO) layer is then deposited on the polysilicon, which serves as the pattern-transfer layer. The LTO $\mathrm{SiO}_{2}$ pattern-transfer layers were also deposited using LPCVD techniques. An LTO $\mathrm{SiO}_{2}$ layer was chosen as opposed to a thermal oxide in order to avoid oxidizing the polysilicon. The slotted cantilever structures are then defined in the polysilicon using a chlorine-based etch $\left(\mathrm{Cl}_{2} / \mathrm{He}\right)$ in order to produce highly vertical side-walls for the capillary slots. Scanning electron micrography (SEM) of the resultant structures revealed that the etched walls were highly vertical in all cases; chlorinebased RIEs are known to produce the relatively high aspect ratio features required for the formation of such microfluidic slots, which are needed here to produce the required capillary action. The triangular cantilevers are released from the silicon wafer surface by wet etching the sacrificial etch release layer of thermal oxide present underneath the polysilicon cantilevers. A $\mathrm{HF}: \mathrm{H}_{2} \mathrm{O}$ solution was used to perform this as the wet etch selectivity between the $\mathrm{SiO}_{2}$ and the polysilicon is very high for concentrated HF solutions. Finally, the cantilevers are made to overhang the edge of the silicon wafer via wafer cleaving along the natural (100) cleavage lines of the silicon. The clamped-free triangular cantilevers can be easily made as long as $1000 \mu \mathrm{m}$.

Furthermore, variations on the micronib theme can be created by applying further microand nanotechnology techniques. Figure 3 shows three such refinements on the idea of a simple capillary slot topology. The first of these variations is termed a 'halfnib' as half of the microtip is removed, allowing the liquid meniscus to wet the remaining half of the nib tip. The second variation is termed a pointnib; here the third dimension of the nib tip has been milled to form a point-like structure (curvature $\sim 100 \mathrm{~nm}$ ) using focused ion beam (FIB) etching. FIB etching can also be used to mill the end of a cantilever structure in order to form what we term a nanonib [18], which comprises a nanofluidic capillary slot. The example shown in the figure measures $50 \mathrm{~nm} \times 300 \mathrm{~nm} \times 4 \mu \mathrm{m}$.

\subsection{Physics of spontaneous capillary slot filling}

We continue by recalling results on the capillary slot filling of a rectangular symmetric slot which we derived earlier [19]. As essential control parameters we identified the normalized slot width $w / h$ (the inverse of the aspect ratio $R$ which is commonly used in microtechnology) and the contact angles $\theta_{\mathrm{s}}$ in the slot, and $\theta_{\mathrm{w}}$ on the outer surface of the slot, which are not necessarily identical due to the fabrication process. The desired liquid structure to fill the slot is an elongated bridge, which preferably builds up in slots with low contact angles and high aspect ratios.

The theoretical determination of liquid shapes is based on the condition of mechanical equilibrium of the liquid-vapour interface, given by the Laplace equation

$$
2 \gamma_{\mathrm{lv}} M=P_{1}-P_{\mathrm{v}},
$$

where

$$
M=\frac{1}{2}\left(c_{1}+c_{2}\right)
$$

is the mean curvature of the interface, expressed through the two principal curvatures. Equation (1) relates the surface tension of the liquid-vapour interface and its mean curvature to the bulk pressure difference between the two fluid phases. The balance of forces induced by the surface tensions $\gamma_{i j}$ with $i, j=\mathrm{v}, 1, \mathrm{~s}$ for the vapour, liquid and solid phases, respectively, is described by the Young-Dupré equation

$$
\gamma_{\mathrm{lv}} \cos \theta=\gamma_{\mathrm{vs}}-\gamma_{\mathrm{ls}}
$$



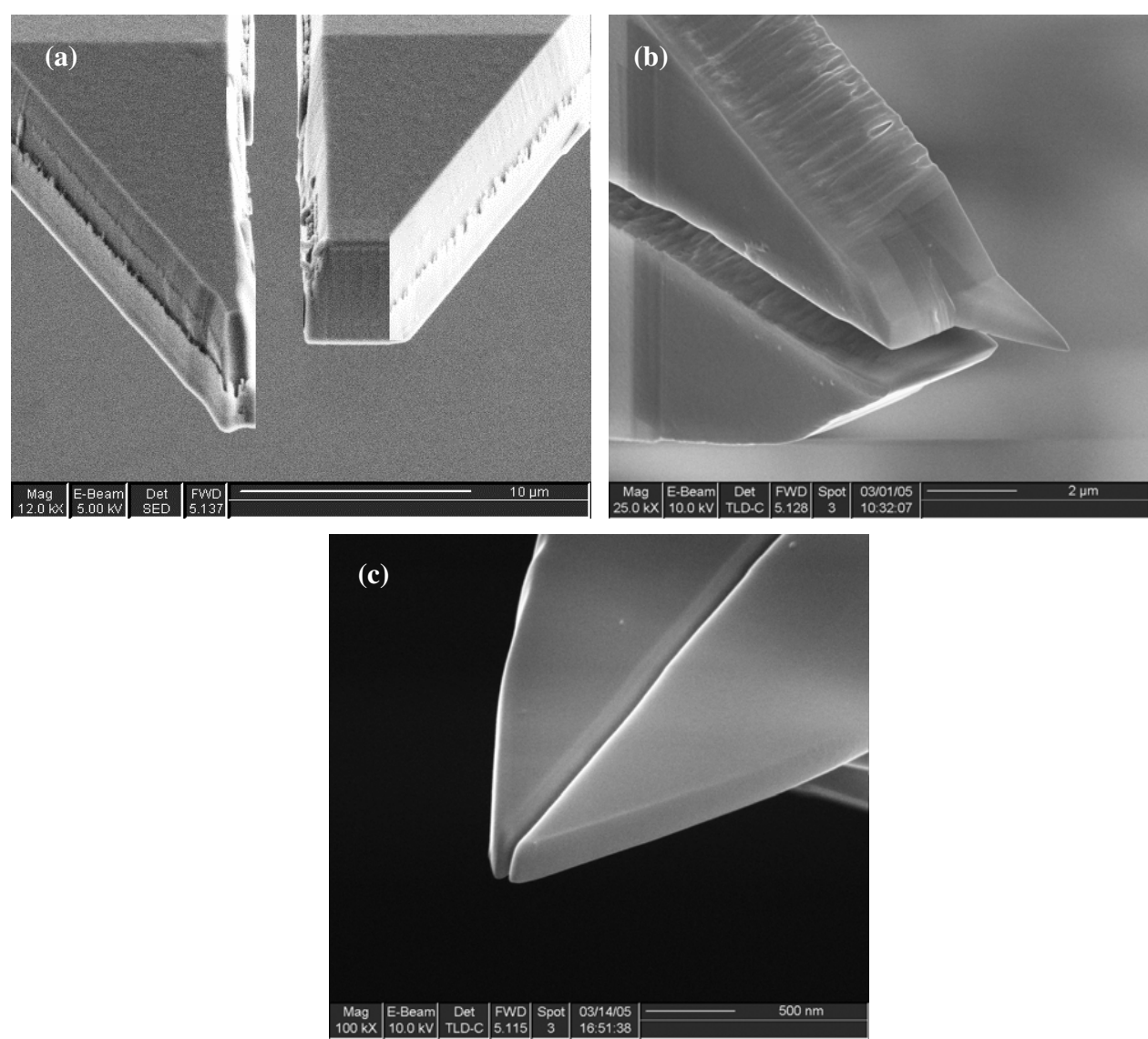

Figure 3. Variations on the micronib theme created using focused ion beam (FIB) nanofabrication: (a) a 'halfnib' and (b) a 'pointnib', both of which use meniscus effects at the end of a slot to 'sharpen' the meniscus of the liquid at the extremity of the tip, and (c) a 'nanonib', which incorporates a nanofluidic capillary slot (the dimensions of the nanofluidic capillary slot $w \times h=50 \times 300 \mathrm{~nm}$ ).

where $\theta$ is the contact angle of the liquid-vapour interface. It should be noted that this equilibrium condition only holds on the smooth parts of the substrate walls; if a contact line arises on a sharp edge of the substrate, the contact angle is ill-defined and can vary in a certain range of values.

Liquid equilibria can be determined numerically by minimization of the interfacial energy, e.g. with the program Surface Evolver [20]. For liquids in geometries of high symmetry, analytic approximations can also be invoked [19]. We summarize our previous findings as follows. Spontaneous imbibition of the liquid requires the existence of elongated liquid bridges with a negative Laplace pressure in the slot. In the case $\theta_{\mathrm{s}}=\theta_{\mathrm{w}}$, such elongated bridges are always found if the conditions

$$
\theta_{\mathrm{w}}<90^{\circ} \quad w / h<1
$$

are fulfilled. Indeed, the optimal range for capillary slot filling is bounded by the line

$$
\frac{w}{h}=\cos \theta_{\mathrm{w}} .
$$

One particular interest in the capillary slot-based electrospray devices lies in the possibility to modify the shape of the liquid meniscus. We have addressed this additional degree of 

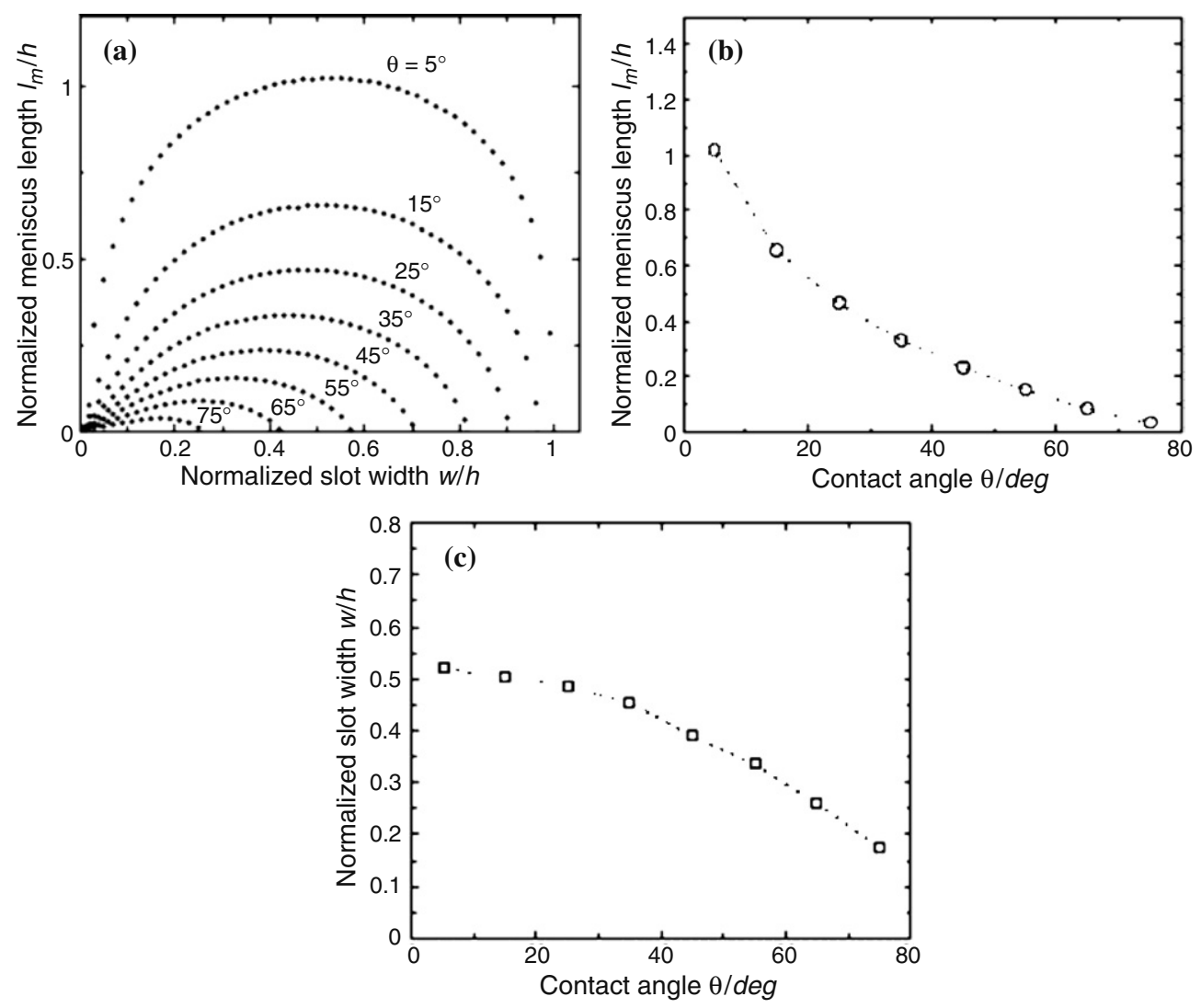

Figure 4. Theoretical predictions for the asymmetric slot: (a) meniscus length as a function of the slot width for various values of the contact angle; (b) maximal meniscus length as function of the contact angle; (c) slot width at maximal meniscus length as a function of the contact angle.

freedom by considering asymmetric slots, in which the two cantilevers defining the slot have different lengths, with the motivation to affect the liquid meniscus; see figure 3.

Naming the difference in extensions of the two slot sides the point length $\ell_{\mathrm{p}}$, the liquid meniscus can be forced to 'reach beyond' the slot up to a meniscus length $\ell_{\mathrm{m}} \leqslant \ell_{\mathrm{p}}$. The question now arises as to what values $\ell_{\mathrm{m}}$ may assume upon variation of contact angles and slot parameters.

Technically, the computation was performed by numerical minimization of the interfacial energies with the program Surface Evolver. Figure 4 summarizes three results we have obtained from these considerations. Figure 4(a) shows the normalized meniscus length $\ell_{\mathrm{m}} / h$ versus the normalized slot width, $w / h$. For the range of permissible contact angles $\theta_{\mathrm{w}}<90^{\circ}$, the meniscus length is found to obey a quasi-circular curve with a maximum at values $0.1<$ $\ell_{\mathrm{m}}^{\max } / h<0.5$. As functions of contact angle, both lengths display a decay towards contact angles of $90^{\circ}$, whereby the meniscus length reduces considerably faster (figures 4 (b) and (c)).

\section{Microfluidic tests}

\subsection{Fabrication of test structures}

In order to perform microfluidic tests to assess the filling of a microfluidic capillary slot by a liquid, we fabricated microfluidic capillary slots using a silicon wafer having a 3 inch diameter. 
Table 1. Measured slot width $w$ and aspect ratio $R=h / w$ for a slot height of $230 \mu \mathrm{m}$.

\begin{tabular}{ll}
\hline Slot width $w(\mu \mathrm{m})$ & Slot aspect ratio $R$ \\
\hline 45 & 5.11 \\
63 & 3.65 \\
87 & 2.64 \\
140 & 1.64 \\
163 & 1.41 \\
266 & 0.86 \\
485 & 0.47 \\
\hline
\end{tabular}

A dry etching technique called 'inductively coupled plasma' (ICP) is used to define the high aspect ratio capillary slots having highly vertical side walls. Firstly, an etch-stop layer must be deposited on the rear side of the silicon wafer in order to avoid unwanted isotropic etching at the bottom of the capillary slots. A layer of silicon dioxide $(2 \mu \mathrm{m})$ is used to achieve this due to its high etch selectivity compared to silicon $(\sim 300)$ for the dry etch process used here (SF6). Next, a hydrofluoric acid based wet etch allows the removal of the silicon dioxide uniquely on the front side of the wafer. Following this, a $15 \mu \mathrm{m}$ thick masking layer (the photoresist AZ9260) is deposited on the front side of the silicon wafer surface in order to locally dry etch the silicon to form the capillary slots. Finally, organic material is removed using acetone and Piranha $\left(\mathrm{H}_{2} \mathrm{SO}_{4} / \mathrm{H}_{2} \mathrm{O}_{2}\right)$ and the silicon dioxide is removed using hydrofluoric acid. All microfluidic slots had a length $L$ equal to $20 \mathrm{~mm}$. The slot height $h$ is defined by the thickness of the semiconductor wafer which was $230 \mu \mathrm{m}$. The slot width $w$ is defined by the photomask. Table 1 shows the slot widths defined by the dry etching procedure described above together with the aspect ratio $(h / w)$ of the capillary slots which varies from 5.11 to 0.47 .

\subsection{Testing the spontaneous filling of a microfluidic capillary slot}

Solutions of de-ionized water and semiconductor grade methanol mixtures were carefully prepared in order to conduct the microfluidic tests. Eleven solutions were prepared by varying the methanol concentration from 0 to $100 \%$ vol:vol in 10\% steps. Different methanol concentrations enable us to vary the wetting contact angle on the slot walls $\theta_{\mathrm{w}}$. The wetting contact angle $\theta_{\mathrm{s}}$ of these solutions was measured on a clean silicon wafer surface; the silicon wafer was n-type $\left(10^{17} \mathrm{~cm}^{-3}\right)$ having a (100) crystal orientation. Prior to the contact angle measurements, the silicon wafer was cleaned using a Piranha solution $\left(\mathrm{H}_{2} \mathrm{SO}_{4}: \mathrm{H}_{2} \mathrm{O}_{2}\right)$ and hydrofluoric acid (HF). The contact angles were measured using a Digidrop EWS contact angle meter (GBX, France) by placing a fixed volume $(0.5 \mu \mathrm{l})$ onto the wafer surface; a good reproducibility of the contact angle was achieved $\left( \pm 5^{\circ}\right)$. The results of the contact angle measurements are summarized in table 2.

In order to observe the spontaneous filling of the capillary slots, a drop $(\sim 1 \mu \mathrm{l})$ of each solution was carefully placed at the end of each capillary slot using a pipette and the spontaneous filling was observed using an optical microscope. Figure 5 shows an image of a solution on one of the microfluidic capillary slots; the wetting angle is clearly seen. The different solutions were used in turn in order to assess whether or not spontaneous filling was present for a slot having a given aspect ratio.

As a note on the observation and characterization of nanofluidic devices let us recall that it is straightforward to perform observational fluidic tests on channels and slots provided that at least two system dimensions are in the micrometre range. In this context, we have previously visualized the spontaneous filling of a microfluidic capillary slot by a liquid using 


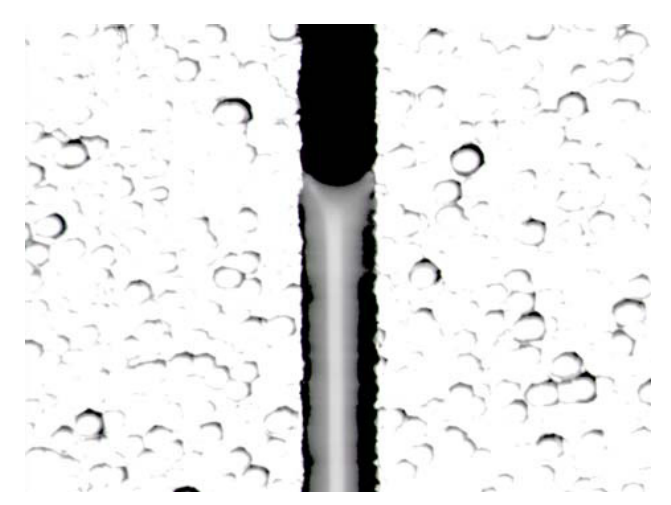

Figure 5. A 50:50 vol.:vol. $\mathrm{MeOH}: \mathrm{H}_{2} \mathrm{O}$ mixture in a micro-machined capillary slot having a width $w$ of $140 \mu \mathrm{m}$ and a height $h$ of $230 \mu \mathrm{m}(R=1.64)$.

Table 2. Wetting contact angle $\theta_{\mathrm{s}}$ of the concentration of methanol:water solutions, on silicon(100). Theoretical minimum aspect ratio $R_{\text {th }}$ which allows spontaneous filling of the slot; observed minimum aspect ratio $R_{\mathrm{ob}}$ for filling.

\begin{tabular}{rccc}
\hline$c(\%)$ & $\theta_{\mathrm{s}}$ & $R_{\mathrm{th}}$ & $R_{\mathrm{ob}}$ \\
\hline 0 & 80 & 5.76 & 5.11 \\
10 & 74.4 & 3.72 & 3.65 \\
20 & 64.2 & 2.30 & 3.65 \\
30 & 59.1 & 1.95 & 2.64 \\
40 & 42.3 & 1.35 & 1.41 \\
50 & 37.5 & 1.26 & 1.41 \\
60 & 31.4 & 1.17 & 1.41 \\
70 & 26.3 & 1.12 & 1.41 \\
80 & 18.7 & 1.06 & 1.41 \\
90 & $<10$ & 1.02 & 0.86 \\
100 & $<5$ & 1.00 & 0.86 \\
\hline
\end{tabular}

optical microscopy [21]. However, we note that the observation of nanofluidic filling of capillary channels and slots having dimensions of $w, h$ and $L$ is much more difficult when $w$ and $h$ are on the nanometre scale and $L$ is of the order of a few micrometres. Optical microscopy observation of a moving meniscus can still be achieved when $L, w \gg h$, where $h$ has nanometre dimensions, say $10 \mathrm{~nm}, w$ has micrometre dimensions and $L$ is on the millimetre scale [22]. The devices presented here have dimensions which are at the limit of these conditions (micronib) or do not fulfil these conditions at all, as the smallest capillary slot may have $w$ and $h$ on the nanometre scale as is the case of the nanonib; one must thus turn to a non-observational characterization, e.g. mass spectrometry.

The spontaneous filling observations, shown in table 2 and figure 6 , are seen to agree well with the theoretical predictions of section 2. However, there are slight differences with the predicted minimum value of $R_{\mathrm{th}}$; this can be explained by the fact that the slot wall surface energy following a dry etch is likely to be different both physically (through an increased surface roughness induced by photomask defects and non-perfect wall-definition during dry etch) and chemically (through deposits of polymers generated during the dry etch) from that of a clean silicon wafer surface; this implies that the wetting contact angle on the slot walls $\theta_{\mathrm{w}}$ will not be equal to $\theta_{\mathrm{s}}$. 

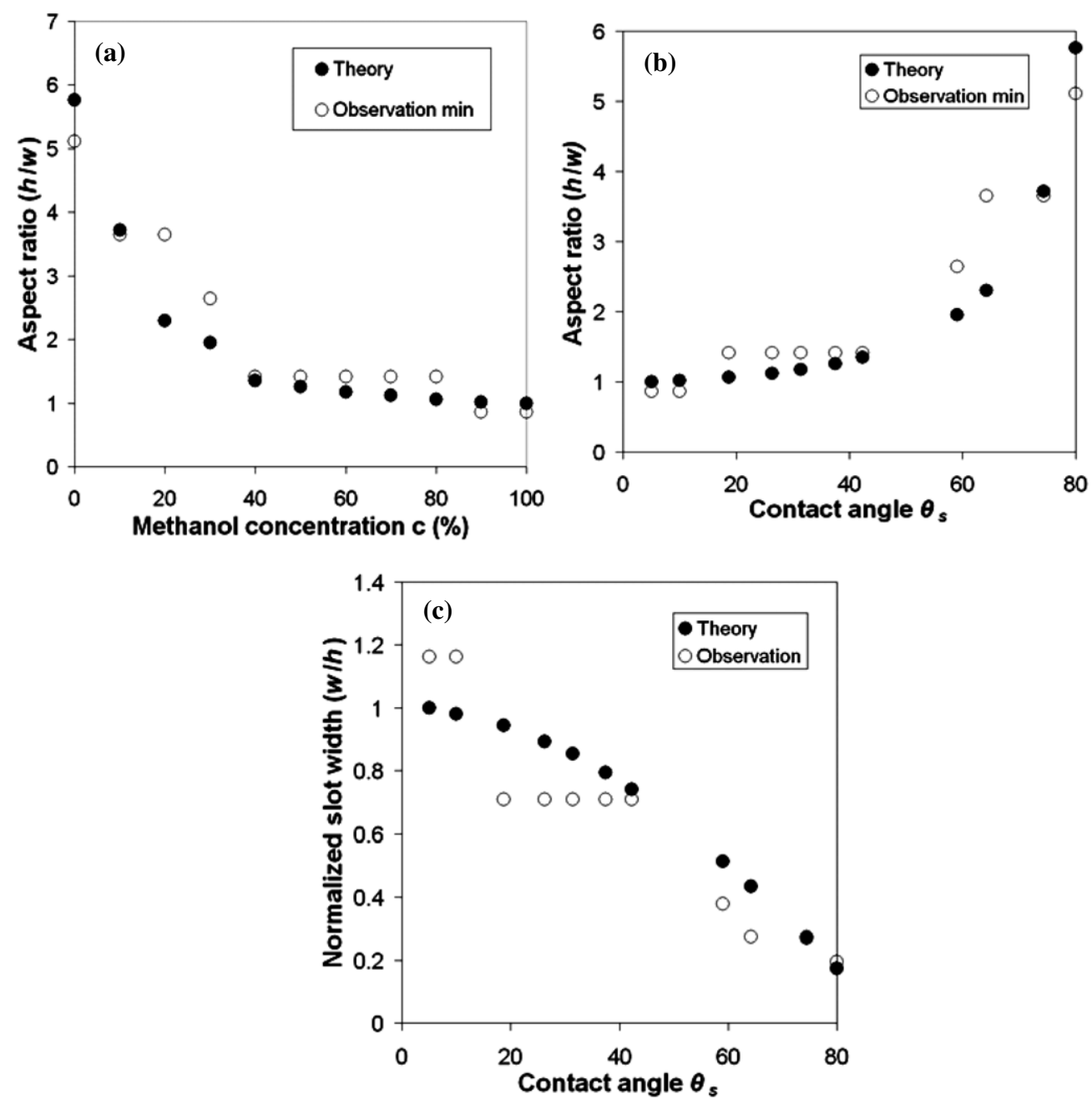

Figure 6. Filled circles show the theoretical minimum aspect ratio required for spontaneous filling as a function of (a) methanol concentration $c$ and (b) contact angle $\theta_{\mathrm{s}}$; open circles show the observed minimum aspect ratio for spontaneous filling; (c) normalized slot width $w / h$ as a function of wetting contact angle.

\subsection{Meniscus overshoot at the end of the capillary slot}

The photomask also incorporated a zone which included test features which enabled us to measure the overshoot of the meniscus at the end of a capillary slot. Figure 7 shows optical images which demonstrate the evolution of the meniscus length as a function of slot width $w$ for a fixed methanol concentration $c$ of $50 \%$ in water. Table 3 shows the results of the microfluidic tests. Finally, figure 8 shows a graphical representation of the microfluidic test results by plotting the normalized meniscus length $\ell_{\mathrm{m}} / h$ as a function of methanol concentration $c$ and normalized slot width $w / h$.

If we compare these results to the theoretical results of section 2 we observe the predicted evolution in the value of normalized meniscus length $\ell_{\mathrm{m}} / h$ as a function of $w / h$ and wetting contact angle $\theta_{\mathrm{w}}$ (defined by the methanol concentration $c$ ). The normalized meniscus 

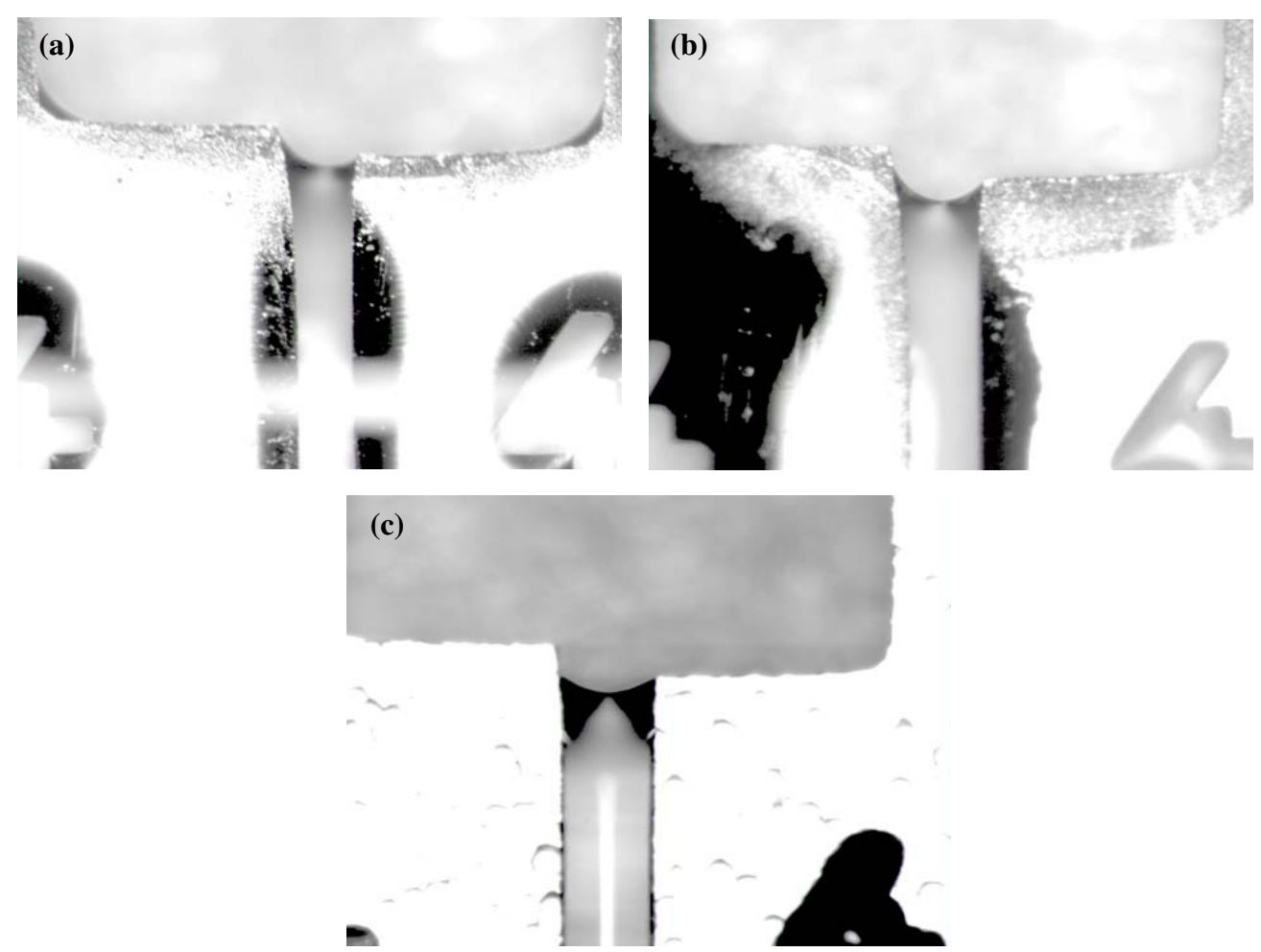

Figure 7. Optical microscopy images of the meniscus overshoot length $l_{\mathrm{m}}$ as a function of slot width $w$ at the end of a microfluidic capillary slot for a methanol concentration $c=50 \%$ in water: (a) $w=130 \mu \mathrm{m}$, (b) $w=160 \mu \mathrm{m}$ and (c) $w=200 \mu \mathrm{m}$.

Table 3. Measured meniscus length $\ell_{\mathrm{m}}$ in $\mu \mathrm{m}$ as a function of slot width $w$ and methanol concentration $c$ vol.:vol. \%.

\begin{tabular}{lllllll}
\hline & \multicolumn{6}{c}{$c$} \\
\cline { 2 - 6 } Slot width $w(\mu \mathrm{m})$ & 30 & 40 & 50 & 60 & 70 & 80 \\
\hline 100 & & 13 & 15 & 19 & & \\
130 & 31 & 53 & 60 & 65 & 70 & 75 \\
160 & & 20 & 32 & 41 & 49 & 52 \\
200 & & & 12 & 27 & 32 & 54 \\
\hline
\end{tabular}

length increases with increasing methanol concentration, i.e. decreasing wetting contact angle. Further, the meniscus length goes through a maximum as a function of varying microfluidic slot width, as shown before. However, it is difficult to obtain the exact value of the maximum normalized meniscus here as we only have four fixed slot widths.

\section{Mass spectrometry tests}

Mass spectrometry tests were performed using a Q-TOF mass spectrometer. The liquid test sample was loaded manually into the source using a micropipette, the liquid sample entering the capillary slot by capillary action and reaching the tip of the source. Upon application of the HV, 

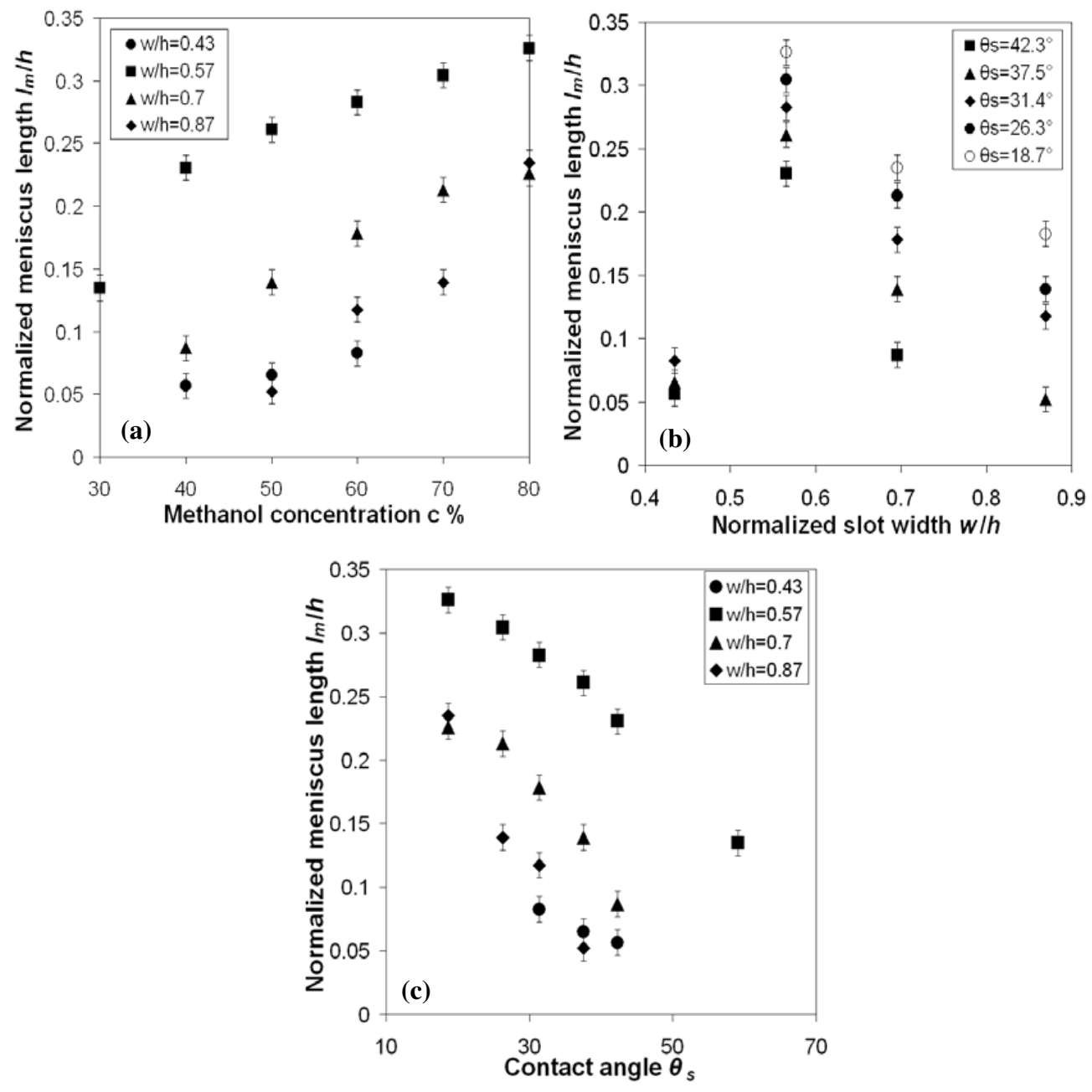

Figure 8. A graphical summary of the meniscus overshoot microfluidic experiments showing the normalized meniscus length $l_{\mathrm{m}} / h$ versus (a) methanol concentration $c$, (b) normalized slot width $w / h$ and (c) wetting contact angle $\theta_{\mathrm{s}}$; error bars on $l_{\mathrm{m}} / h$ are determined to be \pm 0.01 of experimentally determined values.

a Taylor cone forms at the end of the capillary slot of the micronib and electrospraying occurs. As for the standard needle-based electrospray sources the micronib was orthogonal to the input of the mass spectrometer. Figure 9 shows the set-up employed for the mass spectrometry testing.

Initial investigations were performed to see how the micronibs performed in comparison to standard commercial capillary-based needle-like electrospray sources based on capillary tubes. Preparation of the commercial source was performed routinely via manual loading of the test liquid into the capillary tube based ESI source. The liquid test sample $\sim 5 \mu \mathrm{l}$ was also loaded manually into the micronib source using a micropipette, the liquid sample entering the capillary slot where the width $w$ and height $h$ are equal to $1 \times 4 \mu \mathrm{m}$ by capillary action and reaching the tip of the source. A capillary voltage of $1.5 \mathrm{kV}$ and a sample cone voltage of $40 \mathrm{~V}$ were applied, data being acquired over a period of $60 \mathrm{~s}$. Upon application of the HV, a Taylor cone forms 


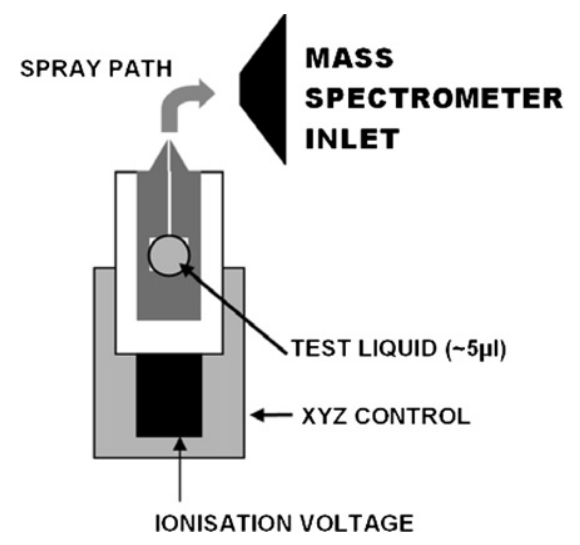

Figure 9. Electrospray configuration used for the mass spectrometry testing.
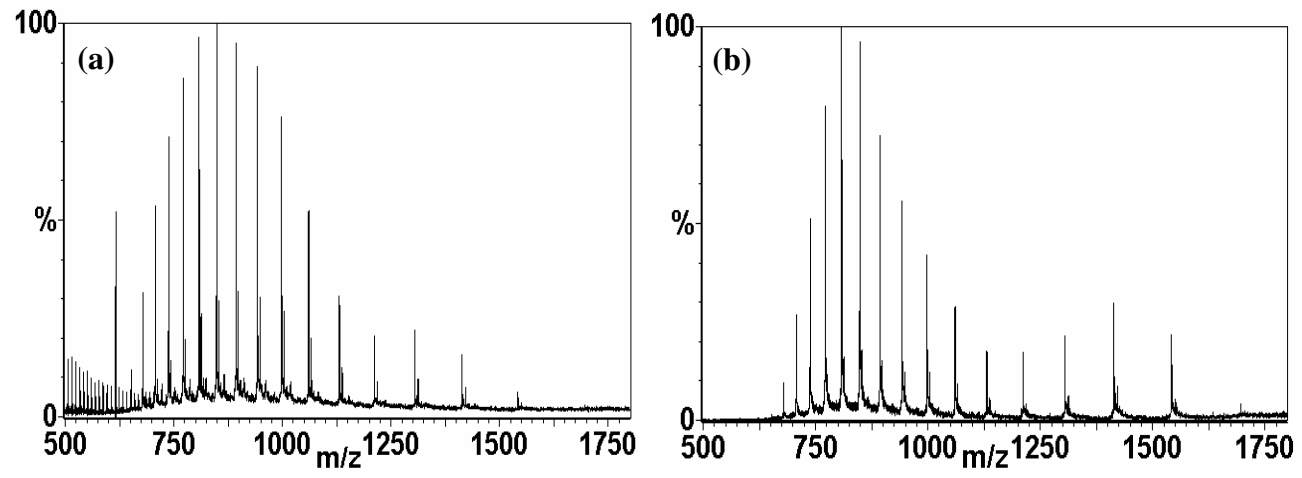

Figure 10. Mass spectra of horse heart myoglobin acquired with a solution of $10 \mathrm{pmol} \mu \mathrm{l}^{-1}$ protein in $1: 1 \mathrm{v} / \mathrm{v}$ water:methanol with $0.5 \%$ formic acid added obtained using (a) a standard capillary tube based needle electrospray source and (b) a polysilicon capillary slot-based emitter tip-MS $w \times h=1 \times 4 \mu \mathrm{m}$.

at the end of the source and electrospraying occurs. As is the case for the standard needlebased electrospray sources, the micronib was orthogonal to the input of the mass spectrometer. Figure 10 shows the set-up employed for the mass spectrometry testing of the micronibs. Figures 10(a) and (b) show mass spectra obtained for horse heart myoglobin acquired with a solution of $10 \mathrm{pmol} \mu \mathrm{l}^{-1}$ protein in $1: 1 \mathrm{v} / \mathrm{v}$ water:methanol with $0.5 \%$ formic acid added using a needle based electrospray source and a polysilicon based capillary slot micronib. The mass spectrum obtained using the polysilicon micronib shows horse heart myoglobin (mass 16951.49 Da) together with a minor phosphate adduct (98 Da higher in mass); however, the singly charged haem residue (mass 615.2 Da) is not present [18].

Initial measurements indicate that the signal-to-noise ratio is higher in spectra using a micro-machined emitter tip, indicating a higher ionization yield. Let us consider the comparison between a polysilicon capillary-based emitter tip and silica-based capillary tube emitters in more detail. Firstly, in the case of a capillary slot, at the end of the tip the liquid will be confined uniquely to the slot due to capillary effects and the lower surface energy of polysilicon for a given liquid. This is in contrast to silica-based tips where wetting of the whole end of the tips can occur due to the high surface energy. The result of confining the liquid 
to the capillary slot is a lower flow rate $Q$ which results in a smaller droplet radius $r$ (since $Q \propto r^{3 / 2}$ ) with a higher surface-to-volume ratio. This leads to an increase in an observed ionization efficiency [8], and hence a more intense spectrum and thus a lower S/N-ratio. This fact shows up in figure 10(b), as compared to (a). The spectrum from the emitter tip is cleaner than that from the commercial gold-plated vial, i.e. there are fewer lower molecular weight contaminants. The emitter tips thus appear ideal for low level detection limits and high S/N spectra.

\section{Discussion and conclusions}

In this paper we have presented results from an ongoing study concerning miniaturized sources for electrospray mass spectrometry. We have conducted a theoretical investigation, based on capillary theory, for the liquid filling of capillary slots and carefully tested the behaviour of the slots in experiment. The agreement between theory and experiment shows that we are able to predict the desirable capillary filling properties of the electrospray sources.

We believe that an improved theoretical understanding is important for the design of miniaturized components for several reasons. In the course of miniaturization, one may enter regimes where physical properties, which otherwise can be neglected in more macroscopic settings, matter. In addition to this, the optimal operating conditions for such devices are not obvious. We believe that micro- and nano-machined emitter tips as we have presented them here will allow more controlled studies of electrospray ionization for mass spectrometry as well as fundamental studies of droplet emission.

On the more practical side, prior testing by simulation may bring down development cycles, since the fabrication of the devices is time consuming; and obviously it may also reduce costs.

Finally, we note that one has to be aware that the use of novel device components also poses important challenges for the interpretation of the measurements. The preliminary electrospray mass spectrometry results which we have presented here indicate the need for systematic studies of the relationship between device topology and spectrometric data.

\section{Acknowledgment}

The authors would very much like to thank David Troadec (Research Engineer at IEMN) for the focused ion beam fabrication.

\section{References}

[1] Zeleny J 1914 Phys. Rev. 369

[2] Zeleny J 1917 Phys. Rev. 101

[3] Chapman C 1937 Phys. Rev. 52184

[4] Dole M, Mack L L and Hines R L 1968 J. Chem. Phys. 492240

[5] Mack L L, Kralik P, Rheude A and Dole M 1970 J. Chem. Phys. 524977

[6] Yamashita M and Fenn J B 1984 J. Phys. Chem. 884451

[7] Meng C K, Mann M and Fenn J B 1988 Z. Phys. D 10361

[8] Fenn J B, Mann M, Meng C K, Wong S F and Whitehouse G M 1989 Science 24664

[9] Wilm M S and Mann M 1994 Int. J. Mass Spectrom. Ion. Process. 136167

[10] Schultz G A, Corso T N, Prosser S J and Zang S 2000 Anal. Chem. 724058

[11] Tang K, Lin Y, Matson D W, Kim T and Smith R D 2001 Anal. Chem. 731658

[12] Kim J S and Knapp D R 2001 Electrophoresis 223993

[13] Yuan C and Shiea J 2001 Anal. Chem. 731080 
[14] Gobry V, Oostrum J, Martinelli M, Rohner T C, Reymond F, Rossier J S and Girault H H 2002 Proteomics 2405

[15] Sjoedahl J, Melin J, Griss P, Emmer A, Stemme G and Roeraade J 2003 Rapid Commun. Mass Spectrom. 17337

[16] Arscott S, Le Gac S and Rolando C 2005 Sensors Actuators B 106741

[17] Troadec D and Arscott S 2005 Nanotechnology 162295

[18] Arscott S and Troadec D 2005 Appl. Phys. Lett. 87134101

[19] Brinkmann M, Blossey R, Arscott S, Druon C, Tabourier P, Le Gac S and Rolando C 2004 Appl. Phys. Lett. 851240

[20] Brakke K 1992 Exp. Math. 877768

[21] Arscott S, Le Gac S and Rolando C 2005 Sensors Actuators B 98140

[22] Tas N R, Haneveld J, Jansen H V, Elwenspoek M and van den Berg A 2004 Appl. Phys. Lett. 853274 